\title{
LINGUISTISCHE PHONETIK
}

Obwohl die Phonetik eines der ältesten sprachwissenschaftlichen Fächer ist, ist ihre Stellung innerhalb der Sprachwissenschaft bei weitem nicht klar und scheint sogar zunehmend unklarer zu werden. Es ist deshalb an der Zeit, sich Gedanken über diese Disziplin, ihre Stellung und ihre Aufgaben zu machen. Letzten Endes geht es darum, der Phonetik den ihr gebührenden Platz innerhalb der Sprachwissenschaft zuzuweisen. Zum anderen taucht die Frage auf, warum die Stellung der Phonetik innerhalb der Sprachwissenschaft periodisch unsicher wird. Es erscheint angezeigt sich zuerst dieser zweiten Frage zuzuwenden, bevor die Aufgaben und Eigenschaften einer linguistischen Phonetik hier skizziert werden.

Die Phonetik ist unzweifelhaft eine Grenzwissenschaft in dem Sinne, da $\beta$ sie an der Grenze vieler anderer Disziplinen liegt. Von den angrenzenden Fächern sind insbesondere zu nennen: die Anatomie und Physiologie, die Akustik, die Psychologie und in den letzten Jahren die Neurophysiologie und die Biochemie. In diesem Sinne ist die Phonetik eine Grenzwissenschaft, weil sie Daten und Kenntnisse der Nachbarwissenschaften braucht und sich in ihrer Theoriebildung bewusst auf deren Befunde stützt und stützen muß. Aus diesem Sachverhalt ist deutlich zu ersehen, $d a \beta$ es innerhalb der Phonetik leicht zu Ubertreibungen in die eine oder die andere Richtung kommen kann, indem ein bestimmter Aspekt mehr als ein anderer betont wird. Solche Zwiespalte gab es schon im Altertum. Die Inder haben vorwiegend artikulatorische Kriterien in ihrer Klassifikation der Sprachlaute angewendet. Die Araber haben ebenfalls physiologische Kriterien und Gesichtspunkte vertreten, aber die Griechen, von denen wir so viele Begriffe übernommen haben, u. a. die Begriffe Vokal und Konsonant, vertraten auditive und funktionelle Kriterien - die Hörbarkeit und den silbenbildenden Charakter, "Silbichkeit". Aus diesen Umständen erklärt sich, daß einige Grundbegriffe der Phonetik wie z. B. Vokal, Konsonant, Silbe nie einheitlich definiert worden sind. In der neueren Zeit gewinnen auch verschiedene Gesichtspunkte bei einzelnen Forschern die Oberhand. Einige sind mehr Physiologen, andere Akustiker oder Spezialisten auf noch engeren Gebieten. Es entsteht daher häufig der Eindruck, als ob die Phonetik ein Mosaik von heterogenen Fakten sei. Ein solcher Eindruck kann bei einer Grenzwissenschaft leicht entstehen. Wir sollten aber das Wort Grenzwissenschaft richtig verstehen, nämlich als eine Wissenschaft die darauf angewiesen ist, zum Teil auf den Daten anderer Wissenschaften aufbauen zu müssen. Aber die Phonetik innerhalb der Sprachwissenschaft ist kein Grenzbereich, sondern ein zentraler Bereich von solcher Wichtigkeit, daß die Sprachwissenschaft ohne sie kaum möglich wäre. Ihr Forschungsbereich ist einheitlich und absolut klar definiert: der Sprachlaut. Die Phonetik ist die Wissenschaft von den Sprachlauten, ihrer Form und ihren Eigenschaften.

Eine solche Definition der Phonetik ist aber nicht frei von gewissen Schwierigkeiten, denn eine einheitliche allgemein akzeptierte Definition des Sprachlautes gibt es gleichfalls nicht. Andererseits ist der Sprachlaut innerhalb der Linguis- 
tik auch eine Erscheinung besonderer Art. Kein anderes Phänomen der Linguistik kann sich in einer Substanz manifestieren. Die Phonetik steht daher auch innerhalb der Linguistik in einer besonderen Stellung, denn sie ist die einzige Disziplin der Linguistik, die in Berührung mit Substanzphänomenen kommt. Die Versuchung ist daher sehr groß, besonders weil die Naturwissenschaften eine so glänzende Entwicklung gezeigt haben, diese Substanzerscheinungen als naturwissenschaftlich zu betrachten und die Phonetik und den Sprachlaut mit derartigen Erscheinungen gleich zu setzen. Dieser Versuchung unterlagen die Gründer der sogenannten Experimentalphonetik, die als neue Wissenschaft dargestellt wurde, die tatsächlich aber auf verhängnisvollen Mißverständnissen beruht.

Die Experimentalphonetik hat sich im letzten Jahrhundert entwickelt und zwar nicht aus der Linguistik, sondern aus den Naturwissenschaften. Der Erfolg der sogenannten exakten Wissenschaften schuf ein Modell, nach dem die Phonetik auch zu einer exakten Wissenschaft gemacht werden sollte, indem sie die neuen experimentellen Methoden und Meßtechniken anwendete. Daß die Phonetik unter den linguistischen Disziplinen die einzige Disziplin war, die diese Methoden anwenden konnte, liegt auf der Hand, denn nur sie hat die Sonderstellung, sich mit Substanzphänomenen zu befassen. Die Mißverständnisse, die dieser Auffassung zugrunde lagen, sind grob umrissen, die folgenden: Erstens ist das Experimentieren von Anfang an falsch interpretiert worden. Es geht vielmehr um Registrieren von Phänomenen. Die Bezeichnung Experimentieren ist erst dann zutreffend, als man in der Lage war, Sprache synthetisch zu erzeugen. Zweitens hat man die Sprache mit den registrierten Phänomenen identifiziert. Man ging sogar so weit, da $\beta$ man sich erhoffte, in den Registrierungen mögliche Entwicklungstendenzen der Sprache entdecken zu können, z. B. Lautveränderungen im Anfangsstadium oder in verschiedenen Graden der Entwicklung. Drittens sah man die Hauptaufgabe im AnsammeIn von Daten, ohne zu bemerken, daß man eine Theorie braucht, um Daten geordnet sammeln und dann auswerten zu können beide Aspekte sind völlig untrennbar.

Diese Fehler sind vielleicht aus der anfänglichen Begeisterung über die nicht erwarteten Leistungen der neuen Methoden zu erklären, aber die Folgen derartiger Utbertreibung waren fatal. Es erschienen viele Arbeiten, die nur aus Zahlen und Tabellen bestanden, vor denen der Linguist hilflos war und nicht wußte, was er damit anfangen sollte. Obwohl es Experimentalphonetiker gab, wie Rousselot, die Linguisten waren und sein wollten, war das Gegenteil ebenso häufig der Fall. Man strebte danach, die Phonetik als unabhängige Wissenschaft zu etablieren, was sie gemäß ihrer Natur nicht sein kann und nicht sein konnte. In dieser Richtung folgte man jedoch dem Weg bis zum Ende und behauptete, daß einige Grundbegriffe der Linguistik wie diejenigen der Silbe und des Sprachlautes nicht existierten oder wenigstens, daß sie sich phonetisch nicht nachweisen liessen. Diesen Weg, den Hjelmslev als den des Nihilismus bezeichnet, gingen unter anderem so bekannte Phonetiker wie Giulio Panconcelli-Calzia und E.W. Scripture. Dabei liegt der Schwerpunkt bei den Forschern nicht immer am selben Ort. Einige leugneten durchaus den Wert der linguistischen Begriffe, aber andere kamen auf eine unendlich hohe Zahl von Sprachlauten - zehntausende oder hunderttausende für jede Sprache oder jedes Individuum, indem der Sprachlaut mit der Realisation identifiziert wurde. 
Es ist kein Wunder, daß die Linguisten nach kurzer Periode anfänglicher Ratlosigkeit sich gegen solche Auffassungen verwahrten. So entstand die berühmte Polemik zwischen Jespersen und Rousselot (Rousselot 1911), aber zahlreicher war die Gruppe derjenigen, die sich passiv verhielten und mit Sorge der Entwicklung zusahen. Eine entscheidende aber verhängnisvolle Wende ist durch Trubetzkoy eingetreten. Er verlangte, daß die Phonetik den Naturwissenschaften zugewiesen werde. Sie sollte sich mit physiologischen und akustischen Phänomenen befassen und könnte daher unabhängig von den Sprachwissenschaften betrieben werden. Sie wäre, wie er sich ausdrückte, eine Sprechaktlautlehre. Als Gegenstück dieser Wissenschaft, verlangte Trubetzkoy eine sprachwissenschaftliche Sprachgebildelehre, die er Phonologie nannte. Die Phonologie sollte die Funktion der Sprachlaute studieren oder dasjenige an ihnen, was in dieser Hinsicht relevant war. Das war der Beginn der unglücklichen Teilung zwischen Phonetik und Phonologie, wobei die Phonetik aus der Sprachwissenschaft ausgeschieden und den Naturwissenschaften zugeordnet wurde. Positiv erwies sich hingegen die Forderung nach dem Studium des Systems. Die Sprachlaute wurden als Einheiten in einem System verstanden und sie standen im bestimmten Verhältnis zueinander. Nur innerhalb dieses Systems konnten sie definiert und ihre Eigenschaften spezifiziert werden. Die Trennung in Phonetik und Phonologie erschien den Linguisten, die nicht zugleich Phonetiker waren, im großen und ganzen annehmbar zu sein. Sie befriedigte aber nicht diejenigen, die zugleich Phonetiker waren. So protestierten Eberhard Zwirner und Eli Fischer-Jørgensen von Anfang an gegen diese nach ihrer Meinung unberechtigte Aufteilung, und Louis Hjelmslev widmete dieser Problematik zwei wichtige Aufsätze, in denen er eine ähnliche Haltung einnahm.

Hjelmslevs wichtiger Aufsatz "Neue Wege der Experimentalphonetik" fand keine große Resonanz, obwohl dort ernsthaft versucht wird, diese gesamte Problematik zu lösen. Der Grund für die geringe Resonanz ist vielleicht der, daß zu dieser Zeit die Polemik über Zwirners Phonometrie sehr lebhaft war und Hjelmslevs Standpunkt in vieler Hinsicht mit Zwirners Auffassung übereinstimmte. Hjelmslev legt großes Gewicht auf die formale Natur der Sprache, aber er geht gleich weiter, indem er behauptet, da $\beta$ die Form nie aus Notwendigkeit an eine Substanz gebunden ist: "Da die Substanz nie mit der Form zusammenhängt, kann auch die Lautsubstanz nicht notwendig mit der Sprache zusammenhängen. Daß die Laute die verbreiteste Ausdruckssubstanz ausmachen, hängt von der Natur des Menschen ab, nicht von der Natur der Sprache" (Hjelmslev 1968a, S. 119). Trotzdem zwingen die Tatsachen Hjelmslev die lautliche Natur der Sprache anzuerkennen: "Nur durch die Substanz kann die Form manifestiert werden; ohne die Substanzen würde die Sprache keine Daseinsmöglichkeiten in der menschlichen Gesellschaft haben" (Ibid., S. 119), und die verbreiteste dieser Substanzen ist die Lautsubstanz. Da es so ist und da, trotz aller Schwankungen, die Realisierungen jedoch durch eine Norm zusammengehalten werden, zieht Hjelmslev die wichtige Schlußfolgerung:

"Die Phonetik bleibt also ein wichtiges Gebiet der Sprachwissenschaft. Es darf aber nie aus den Augen verloren werden, daß die Phonetik die Lehre von den Sprachlauten ist. Die Aufgabe der Phonetik ist zu beschreiben, wie die Ausdrucks- 
einheiten eines gegebenen Sprachsystems in einer gegebenen Gesellschaft $\mathrm{zu}$ einem bestimmten Zeitpunkt ausgesprochen werden. Diese Aufgabe setzt die Einheiten der Norm voraus und kann ohne Kenntnis dieser Einheiten nicht gelöst werden. Die Phonetik muß als eine empirisch-deduktive Wissenschaft aufgestellt werden: die sprachlichen Formen mu $\beta$ sie als gegebene Größen hinnehmen und auf dieser Grundlage ihre Manifestierungen und ihre Varianten untersuchen" (Hjelmslev 1968a, S. 120).

Dieses Zitat bildet schon den Kern der Richtung einer linguistischen Phonetik. Die Problemstellung mu $\beta$ auf Grundlage der Sprache bestimmt werden, und zwar aufgrund der Sprache als Form. Daraus folgt, daß eine unabhängige, naturwissenschaftliche Phonetik, wie sie etwa Trubetzkoy gefordert hatte, nicht möglich ist. Eine solche Wissenschaft ist sogar theoretisch nicht möglich. Sie wäre keine Phonetik, sondern eine reine Wellenlehre oder eine reine Physiologie. Trotzdem muß betont werden, daß kein Mittel gescheut werden sollte, um so genaue Kenntnis der Sprachlaute wie möglich zu bekommen, aber die Problemstellung muß sprachlich sein, denn nur so wird und kann die Experimentalphonetik für die Sprachwissenschaft nützlich und sogar unentbehrlich werden. "Die Experimentalphonetik muß einmal Experimentalphonematik werden", ist Hjelmslevs Schlußsatz in seiner tiefgreifenden Analyse.

In diesem Punkte scheint es mir wichtig zu betonen, daß die hier erörteten Probleme bei vielen Linguisten nicht einmal angedeutet werden. So ist die Phonetik bei Pike, Bloomfield, Hockett und den Grammatikern der vergleichenden Grammatik des 19. Jh. ein voll integrierter Teil der Linguistik und sie nimmt ihren Platz unter den anderen linguistischen Disziplinen ein. Diese Linguisten schneiden aber das Problem der Lautsubstanz kaum an, obwohl auch sie gelegentlich artikulatorische Beschreibungen anwenden. Unser Problem betrifft aber die Lautsubstanz als die einzige Manifestation der Sprache im physikalischen Bereich. In diesem Sinne stellen wir fest, daß die Lautsubstanz die einzige mögliche Form einer gesprochenen Sprache ist und daher stellt sich die zwingende Frage nach der Integration dieser Substanz in die Linguistik und in die linguistische Theorie. Diese Frage ist durchaus aktuell, denn die Behauptungen der neveren prädiktiven Phonetik zielen darauf hin, die Natur der phonologischen Systeme nur auf Grundlage der Substanz zu erklären und die Form dabei völlig auszuschließen oder ihre Rolle wesentlich zu reduzieren. Am deutlichsten hat der schwedische Phonetiker Björn Lindblom diese Prinzipien formuliert. Wir werden das Problem erörtern, ob ein solches Verfahren überhaupt möglich ist.

Ausgehend davon, daß die Phonetik die Wissenschaft von Sprachlauten ist und $\mathrm{d} a \beta$ eine von der Sprachwissenschaft losgelöste Phonetik ein Unding wäre, will die moderne linguistische Phonetik versuchen, die Erkenntnisse der experimental-phonetischen Forschungen voll und ganz in eine sprachwissenschaftliche Theorie zu integrieren. Es handelt sich aber noch nicht um eine vollentwickelte Wissenschaft. Bis heute ist man erst dahin gelangt, daß allgemeine Leitlinien formuliert worden sind. Da diese Richtlinien jedoch wegweisend sind, ist es angezeigt, sie hier genauer zu erläutern. 
Wichtig ist jedoch dies zu betonen: Die linguistische Phonetik versteht sich nicht als eine isolierte Wissenschaft, sondern aIs einen integrierten Teil der Linguistik. Sie leugnet nicht die interdisziplinären Beziehungen zu pädagogischen, medizinischen und nachrichtentechnischen Fächern und sie verzichtet keineswegs darauf die technischen Errungenschaften im Bereich der Elektronik und der Nachrichtentechnik anzuwenden. Sie sieht aber die Probleme der technischen Seite und diejenigen der Registrierung nicht als ihre Hauptprobleme an. Die Hauptprobleme der linguistischen Phonetik sind vielmehr die Interpretation und nicht das Ansammeln der Daten. Daher geht die linguistische Phonetik von einigen Grundgedanken aus, die folgendermaßen formuliert werden können:

1. Der Grundgedanke der linguistischen Phonetik ist der Gedanke der Opposition. Phonologische Einheiten werden nicht als funktionelle (d.h. relevante) phonetische Einheiten angesehen, sondern als linguistische Einheiten. Linguistisch bedeutet hier, daß diese Einheiten innerhalb einer Sprache auftreten. Daß diese Einheiten nicht phonetisch funktionell sind, bedeutet, $\mathrm{da} \beta$ die phonetische Substanz an sich die Funktion der Einheit nicht bestimmt. Die Folgen dieses ersten Grundsatzes sind außerordentlich wichtig:

a) Keine phonologische Kategorie - sei es der Akzent, die Silbe, das Phonem kann aufgrund spezifischer phonetischer Kriterien definiert werden. Diese Kategorien werden ausschließlich aufgrund von Oppositionen definiert und diesen oppositionsbildenden Einheiten werden phonetische Parameter zugewiesen.

b) Das Phonem wird also nicht vom Sprachlaut der traditionellen Phonetik abgeleitet, sondern umgekehrt - der Sprachlaut wird vom Phonem abgeleitet. Daraus folgt, daß der Sprachlaut nur als eine Variante, oder besser, als die Variante eines Phonems existieren kann. Es folgt daraus ferner, da $\beta$ weder die experimentelle Phonetik überhaupt als selbständige Disziplin möglich ist. Sie kann nur als angewandte Phonemtheorie existieren, wenn sie Phonetik sein will. Eine phonologische Analyse (die auch unbewupt durchgeführt werden kann) ist die Voraussetzung einer phonetischen Analyse. Diese Haltung bedeutet eine Kehrwendung gegenüber der Haltung der Prager Schule, die die beiden Disziplinen trennte. Nach der heutigen Auffassung ist eine Trennung der beiden Disziplinen nicht nur nicht wünschenswert, sondern theoretisch unmöglich, zumal die Abhängigkeit einseitig ist. Phonetik kann nur als angewandte Phonemtheorie getrieben werden. Nur auf dieser Grundlage, so ist die Ansicht der Befürworter der linguistischen Phonetik, kann der Phonetik der ihr gebührender Platz innerhalb der Linguistik angewiesen werden.

2. Der zweite Grundsatz, den man vielleicht eher eine Feststellung nennen könnte, ist der, daß gewisse Eigenschaften oppositiver Einheiten automatisch sind, d.h. von der Umgebung oder von der Physiologie abhängig. Automatische phonetische Phänomene sind nicht bewußt kontrollierbar, und können daher nicht als Unterscheidungsmerkmale oppositiver Einheiten auftreten. Solche automatische Eigenschaften müssen in der Analyse berücksichtigt werden, denn sonst wäre eine Abstraktion von der Substanz nicht möglich. Eine gewisse Vorsicht ist jedoch in der Interpretation der Daten geboten, denn in einer bestimmten Umgebung kann eine gewisse Eigenschaft distinktiv sein, aber in einer anderen Umge- 
bung kann vielleicht dieselbe Eigenschaft nicht distinktiv sein. So ist die Interpretation jedesmal verschieden, auch wenn das physiologische oder das akustische Phänomen in beiden Fällen dasselbe ist.

3. Phonologische Einheiten sind hörbar verschieden und unterschiedbar. Es kann aber oft schwierig sein, z. B. im Bereich der Intonation, diese Einheiten in Verbindung mit lexikalischen, morphologischen oder semantischen Unterschieden zu setzen, aber prinzipiell ist es immer möglich. Die Tatsache, daß alle phonologischen Einheiten hörbar verschieden sind, ist jedoch eine ausreichende Bedingung, um sie für eine Analyse geeignet zu machen.

Aus diesen drei Grundsätzen folgt, daß die Daten der linguistischen Phonetik im Prinzip weder meßbar noch registrierbar sind. Denn die Daten der linguistischen Phonetik sind die Kontraste und Oppositionen, die in den Sprachen der Welt auf der systematisch-phonetischen Ebene beobachtet werden können. Meßbar sind nur die Manifestationen dieser Oppositionen und Kontraste in der Substanz. Aufgrund der regelhaften Beschreibung der vorkommenden Kontraste und deren Manifestationen kann man zu Inventaren über die physiologischen bzw. akustischen Korrelate der Oppositionen kommen. Der umgekehrte Weg ist aber nicht möglich: von den artikulatorischen Stellungen oder der akustischen Registrierung können wir nicht zu Aussagen über das Lautsystem einer Sprache kommen. Deshalb sind keine experimentell registrierten Daten interpretierbar, ohne vorher den Text zu kennen.

Hier entsteht ein delikates und noch ungelöstes Problem. Es wird klar geworden sein, daß in der linguistischen Phonetik die Form die absolute Priorität der Substanz gegenüber besitzt. Das bedeutet, daß wir nur Oberflächenphänomene als Lautmanifestation registrieren können und daher stellt sich zwangsläufig die Frage nach der Verbindung des Oberflächenphänomens zu der zugrundeliegenden Repräsentation. Es wäre übertrieben zu behaupten, daß hier eine allgemeingültige Lösung in Sicht ist, aber die Probleme können entschärft werden, wenn man die Realisierung nur innerhalb des betreffenden Sprachsystems betrachtet. Man darf die Manifestationen nicht nach dem System des Forschers betrachten, sondern als Bestandteil eines geschlossenen Systems. So sind (e $\epsilon$ ) im Grönländischen ein $[i]=/ i /$, aber nicht im Deutschen und anderen germanischen Sprachen. Obwohl man heute nicht weiß, wie die Oberflächenrealisierung der zugrundeliegenden Repräsentation eindeutig zugeordnet wird, bleibt doch die Tatsache, daß die linguistische Phonetik in der Lage sein muß, alle in der Oberflächenstruktur vorkommenden Realisierungen zu beschreiben. Das bedeutet wiederum, daß man es mit realen Phänomenen zu tun hat, die auf keinen Fall vernachlässigt werden dürfen. Man muß sorgfältig vermeiden, sich durch eine phonologische Ideologie so blenden zu lassen, daß man beobachtbare Phänomene entweder vernachlässigt oder gar deren Existenz leugnet. Mit einer solchen Haltung wird der Wissenschaft nicht gedient, sondern sie führt zu starren Fronten, die der Entwicklung der Wissenschaft nur Schaden zufügen können, indem sie jedes Gespräch zwischen den Wissenschaftlern, die unterschiedliche Richtungen vertreten, unmöglich macht. 
Die Ziele der linguistischen Phonetik sind im wesentlichen als die Beschreibung eines Inventars von distinktiven Merkmalen definiert worden. Als Versuch in diese Richtung sind die 26 von Ladefoged vorgeschlagenen Merkmale anzusehen, die alle phonetisch motiviert sind, um die Probleme zu vermeiden, die entstehen, wenn man mit phonetisch nicht motivierten Merkmalen operiert. Merkmale solcher Art sind einige der Merkmale von Jakobson, Fant, Halle wie kompakt/diffus, deren Interpretation und Definition in erster Linie subjektiv bleibt.

Mir scheint jedoch das Ziel zu eng gefasst, wenn es auf die Aufstellung dieser distinktiven Merkmale beschränkt bleibt. Es ist nämlich wichtig, die genaue $\mathrm{Be}-$ schreibung der oppositiven Einheiten zu kennen. So existiert z. B. im Schwedischen ein [5], das eine recht spezielle Artikulation hat, jedoch keine Opposition zu [S] bildet. Beide könnten daher durch dieselben Merkmale beschrieben werden, aber damit wäre nur wenig erreicht, denn wir würden damit die Realisierung der Opposition nicht kennen. Dazu brauchen wir als den zweiten Schritt eine genaue phonetische Beschreibung, nachdem die Existenz einer Opposition festgestellt worden ist. Die Beschreibung und Kenntnis der Substanz bleibt daher ein wichtiger Punkt in der linguistischen Phonetik, auch wenn es nur ihre zweite Aufgabe nach der Feststellung der vorhandenen Oppositionen ist.

Mir scheint in diesem Zusammenhang zweckmäßig auf die Thesen der prädiktiven Phonetik einzugehen. Die prädiktive Phonetik wird heute als eine neue phonetische Richtung dargestellt. Das Prinzip ist aber nicht neu, denn der französische Phonologe André Martinet hat die Prinzipien in seinem bekannten Werk "Economie des changements phonétiques" bereits formuliert und angewendet: das Prinzip der Asymetrie der Artikulationsorgane, das Prinzip des maximalen Kontrastes und das Prinzip der Trägheit der Artikulationsorgane. Lindblom und seine Mitarbeiter haben sich besonders auf die Untersuchung des Prinzips des maximalen Kontrastes konzentriert und es ist unzweifelhaft ihr größtes Verdienst gewesen, diese Begriffe experimentell untersucht $\mathrm{zu}$ haben. Lindblom versteht seine Theorie als erklärende Phonologie (explanatory theory of phonology), aber er gibt zu dieser keine endgültige Definition, sondern skizziert lediglich den Aufgabenbereich der Disziplin, die solche Phänomene wie Lautveränderungen, Lautsysteme (Vokale und Konsonanten eines Systems), Kontraste bzw. Oppositionen, Silbenstruktur oder Ursprung der Regeln, Regelreihenfolge (rule ordering), natürliche Klassen und Merkmale erklären sollte. Es könnte vielen zweifelhaft erscheinen, ob eine so umfangreiche Theorie möglich ist. Davon abgesehen, interessiert uns hier in erster Linie, wie dieses Ziel erreicht werden soll. Wir stellen deshalb erstmal die Frage zurück, ob es überhaupt erreicht werden kann.

Lindblom hat in langjähriger Arbeit Modelle des Sprecherzeugungs-apparates entwickelt und es ist ihm gelungen, auf der Grundlage dieser Modelle gewisse Eigenschaften der Substanz der Sprachlaute vorauszusagen. Daher kommt der Name dieser Richtung "prädiktive Phonetik". Diese Theorie versteht sich als substanzgebunden, wie es aus den Ausgangshypothesen zu sehen ist und die so lauten:

1. Die Priorität der linguistischen Form muß̧ bezweifelt werden. 
2. Wenn nun einmal diese Priorität zurückgewiesen worden ist, kann die Lautstruktur vorwiegend prädiktiv aufgrund einer phonetischen Theorie erklärt werden.

3. Eine mehr substanzorientierte Theorie würde dazu dienen können, die Natur und Entwicklung von Lautveränderungen und Lautsystemen zu erklären.

Nach Lindblom sollte eine nach These 2 aufgebaute Phonetik dazu benutzt werden, die phonetischen Ursachen zu explizieren, die den Ursprung der phonologischen Struktur erklären. Man stellt also die Frage nicht nach den phonetischen Korrelaten oder nach der psychologischen Realität der phonologischen Elemente, sondern fängt von der anderen Seite an. Man stellt Hypothesen für die Vorbedingungen der sprachlichen Kommunikation und deren Entwicklung auf, und versucht auf dieser Grundlage zu Aussagen über die phonologische Struktur zu gelangen. Die dritte These ist identisch mit der Fragestellung: ist eine Erklärungsphonologie (explanatory phonology) möglich?

Wenn man diese Thesen und die Argumentationsweise Lindbloms betrachtet, ist man gezwungen festzustellen, daß hier nichts wesentlich Neues vorliegt, sondern lediglich eine Neuformulierung alter Gedanken. Was hier vorgeschlagen wird, ist letzten Endes die Sprache mit der Substanz zu identifizieren, d.h. hier wird der Fehler der älteren experimentellen Phonetik wiederholt. Obwohl Lindblom heute eine große Autorität in der Phonetik ist, ist es absolut notwendig sich $\mathrm{zu}$ fragen, ob ein solcher Weg überhaupt möglich ist. Insbesondere scheint mir die erste These angreifbar. Denn an der Priorität der linguistischen Form zu zweifeln, bedeutet nichts weniger als zu behaupten, daß eine unabhängige nur substanzbezogene Phonetik existieren kann. Man soll also von der Substanz der Schallwellen und der Artikulationsbewegungen zur linguistischen Form gelangen. Die Hypothese kann auf ersten Blick interessant erscheinen, aber bei genauerer Betrachtung scheint sie mir nicht vertretbar zu sein. Mir scheint die Fragestellung irreführend, denn wie kann man etwas voraussagen, wenn man nicht weiß, ob es existiert? Sprache ist ein metaphysisches Phänomen und kein physikalisches. Daß Vokale, Konsonanten, Silben und Lautsysteme überhaupt existieren, wissen wir nur, weil sich in gewissen Substanzerscheinungen eine linguistische Form manifestiert. Erst wenn wir diese Entdeckung gemacht haben, ist es möglich aufgrund der Kenntnis der Anatomie des Menschen und der Gesetze der Perzeption, einige Eigenschaften der Lautstruktur und der Struktur des Signals vorauszusagen. Ohne zu wissen, daß sich hinter der Substanzerscheinung eine Form verbirgt, könnten wir aufgrund der lautlichen Erscheinung allein wahrscheinlich so gut wie gar nichts über den Produktionsmechanismus sagen, geschweige denn überhaupt etwas über die Funktion dieser Signale.

Es soll hier noch einmal daran erinnert werden, daß Funktion kein substanzbezogener Begriff ist, sonderm ein formbezogener - $d$. h. linguistischer. Ohne die Form zu kennen, sind daher nicht die elementarsten Begriffe wie Vokal, Konsonant, Silbe, Funktion, Opposition usw. feststellbar. Eine prädiktive Phonetik, so wie Lindblom sie vorschlägt kann nur als zweiter Schritt der Analyse erreicht werden. Der erste Schritt ist eine phonologische Analyse. Man darf sich nicht 
dadurch verwirren lassen, daß dieser erste Schritt oft unbewußt gemacht wird und auch nicht dadurch, daß wir zur linguistischen Form nur durch ihre Manifestation im Substanzbereich gelangen können.

Mit dieser Einschränkung - daß die prädiktive Phonetik als der zweite Schritt der Analyse erscheint - ist sie eine durchaus mögliche Forschungsrichtung. Es stellt sich aber noch eine nicht weniger wichtige Frage: wo sind die Grenzen einer solchen Phonetik? Diese Frage kann am besten mit einem Beispiel beantwortet werden. Wie bekannt haben alle germanischen Sprachen es gemeinsam, da $\beta$ die Verschlußlaute $\mathrm{p} \underline{\mathrm{t}} \underline{\mathrm{k}}$ nach $\underline{\mathrm{s}}$ (oder anderen stimmlosen Frikativa) ohne Aspiration erscheinen. Man sagt sparen, steif, Sklave gegenüber Paar, tief, Kohle (oder im Isländischen spara, stífux, skapa gegenüber par, tími, kapa). Diese Erscheinung gilt aber nur, wenn es sich um dasselbe Wort handelt. Wenn eine Wort-oder Morphemgrenze vorhanden ist, wird die Aspiration beibehalten: ístak, laus tími, hás karl; was tun? das Tier usw. In einigen kleinen Dialekten des siidamerikanischen Spanischen, wie es in Kolumbien gesprochen wird, kommt auch eine Aspiration nach [s] vor. Eine solche Aspiration ist im romanischsprachigen Gebiet eine Ausnahmeerscheinung, weil die Aspiration im Sinne der germanischen Sprachen dort völlig unbekannt ist. Man kann sich vorstellen, daß es sich hier um die Wirkung eines Substrats handelt, aber das bleibt hypothetisch und für die hier gestellte Frage auch unwichtig. Wichtig ist, daß eine prädiktive Phonetik in der Lage ist, zwei Formen glottaler Aktivität für Aspiration nach [s] vorauszusagen: entweder eine Offnung in der zweiten Phase der Verschlußphase der Verschlußkkonsonanten, oder eine Offnung für jeden Konsonanten. Hingegen bleibt in der Analyse der Unterschied in der Aspiration nach [s] in den germanischen Sprachen, wo sie an eine Morphemgrenze gebunden ist, und in den spanischen Dialekten Kolumbiens, wo sie von der Morphemgrenze völlig unabhängig ist, völlig verborgen.

Eine substanzbezogene Phonetik, die die Priorität der linguistischen Form bezweifelt, könnte hier keinen Unterschied in den Registrierungen und Kurven der beiden Sprachen finden (ganz davon abgesehen, daß sie nie zu dem Sprachlaut als Minimaleinheit gelangen könnte).

Dieses Beispiel zeigt die Grenze einer prädiktiven Phonetik. Diese Grenze wird durch die linguistische Form bestimmt. Die linguistische Form bestimmt daher den Forschungsbereich der Phonetik verstanden als die Wissenschaft von Sprachlauten; und die Grenze der phonetischen Forschungsmöglichkeit wird ebenfalls durch die linguistische Form bestimmt. In einer ernsthaften Forschung ist daher an der Priorität der linguistischen Form kein Zweifel möglich. Eben diese Priorität der Form macht die Phonetik zu einer linguistischen Disziplin ersten Ranges, die daher von der Linguistik nicht unabhängig sein kann. Ladefoged hat sich einmal so ausgedrückt: "Much to the discomfort of some phoneticians (and some linguists), phonetics is not a science that linguistics must presuppose" (Linguistic Phonetics, Working Papers in Phonetics 6, 1967, S. 57, UCLA; zitiert nach Lindblom 1972, p. 65). Diese Bemerkung ist sehr wichtig: Die Phonetik setzt die Linguistik voraus, nicht aber das Gegenteil. Es handelt sich daher um eine einseitige Abhängigkeit: Phonetik ohne Linguistik kann nicht existieren. 
Nachdem der mutige Versuch Lindbloms, eine substanzbezogene Phonetik zu konstruieren, meiner Ansicht nach, als gescheitert angesehen werden kann, mag es so erscheinen, als ob man zu der Ansicht neigt, daß die Phonetik letzten Endes der Linguistik wenig anzubieten hat. Man darf hier nicht übertreiben. Die Phonetik ist nur ein Teil der Linguistik, aber eben ein besonders wichtiger Teil. Sie ist die einzige linguistische Disziplin, die mit Substanzphänomenen in Berührung kommt und daher wirkt die phonetische Wirklichkeit als Fenster zu anderen linguistischen Disziplinen in so weit, als sie in Berührung mit der lautlichen Struktur kommen. Das ist besonders klar im Falle von Sprachen, die nie geschrieben worden sind. Die Phonetik stellt daher einen privilegierten Bereich dar. Sie gibt uns Daten über das Verhalten der Sprecher im Sprachgebrauch in die Hand und ist die einzige Disziplin, in der man die Praxis der Sprache unmittelbar studieren und beobachten kann. Andererseits ist es die Phonetik, mittels derer man die linguistischen Theorien überprüfen und testen kann.

Keine andere Disziplin nimmt eine solche Sonderstellung innerhalb der Linguistik ein. Deshalb ist und bleibt die Phonetik ein zentraler und unentbehrlicher Teil der Linguistik. Wir können Hjelmslev nicht zustimmen, daß die' Lautsubstanz an und für sich nicht etwas wesentliches ist. Die menschliche Sprache kann sich nur durch die Lautsubstanz manifestieren und alle anderen Manifestationen sind sekundär und davon abgeleitet. Daher bleibt die Phonetik ein zentraler Bereich der linguistischen Forschung und wird es auch in der Zukunft bleiben. Ohne die Lautsubstanz hätte die menschliche Sprache sich nie als Kommunikationsmittel konstituieren können. Wir wissen, was die Phonetik für uns bedeutet, wenn wir vor einer nicht mehr gesprochenen Sprache wie z. B. Latein, Tocharisch oder Hethitisch stehen. Daher sollte keine Mühe gescheut werden, so genaue Kenntnis wie möglich von der Lautsubstanz zu erhalten, sich dessen jedoch erinnernd, daß es sich dabei um Beiträge zur Linguistik handelt und nicht um ein Ziel in sich selbst. Die trockenen Daten, die die Experimentalphonetik sammelt, gewinnen erst ihre Relevanz, wenn sie im linguistischen Zusammenhang gesehen werden. Unter solchem Gesichtspunkt müssten sie jedem Linguisten als wünschenswert und willkommen erscheinen, denn der wahre Linguist hat an allen Aspekten und Manifestationsbereichen der Sprache Interesse. Es ist daher unsere Hoffnung, da $\beta$ die Linguisten davon überzeugt werden, da $\beta$ eine substanzgebundene der Form untergeordnete und in ihrer Fragestellung linguistisch orientierte Phonetik ein unentbehrlicher Teil der Linguistik ist. Eine noch nicht in Einzelheiten ausgearbeitete linguistische Phonetik will dieses Ziel erreichen und somit die Verbindung zur Linguistik eindeutig festlegen. Es hängt von dem Erfolg dieser Aufgabe $a b$, ob die Phonetik nun endlich eine klar definierte Stellung innerhalb der Linguistik einnehmen oder ob ihre Stellung weiterhin periodisch unsicher und umstritten bleiben wird.

\section{BIBLIOGRAPHIE}

Alarcos, Maximiliano A. (1925): Precedentes islámicos de la fonética moderna, in: Homenaje a Menéndez Pidal, vol. III, S. 281-308 (Editorial Hernando, Madrid. 
Fischer-Jørgensen, Eli (1941): Phonologie, Arch. für vergl. Phonetik 5, 170-200 (Nachdruck in Georg Heike (Hrsg): Phonetik und Phonologie, S. 60-98 (Fink Verlag, München 1974).

Hjelmslev, Louis (1968a): Neue Wege der Experimentalphonetik. In: Phonometrie II, S. 112-158 (Karger, Basel).

Hjelmslev, Louis (1968b): Uther die Beziehungen der Phonetik zur Sprachwissenschaft. In: Phonometrie II, S. 159-177 (Karger, Basel).

Ladefoged, Peter (1972): Preliminaries to linguistic phonetics (University of Chicago Press, Chicago, 2. Aufl.).

Lindblom, Björn (1972): Phonetics and the description of language Proc. $7^{\text {th }}$ int. congr. phon. sci. Montréal 1971, S. 63-97 (Mouton \& Co., The Hague).

Martinet, André (1955): Économie des changements phonétiques (Francke Verlag, Bern).

Panconcelli-Calzia, Giulio (1948): Phonetik als Naturwissenschaft (Wissenschaftliche Editionsgesellschaft, Berlin).

Pilch, Herbert (1975): Advanced Welsh phonemics, Zeitschrift für celtische Philologie 34, 60-102.

Rousselot, P.J. (1891): Les modifications phonétiques du langage étudiées dans le patois d'une famille de Cellefrouin (Charente) (Welter, Paris).

Rousselot, P.J. (1897-1908): Principes de phonétique expérimentale (Welter, Paris).

Rousselot, P. J. (1911): La phonétique expérimentale jugée par M. Jespersen, Revue de Phonétique 1, 105-113.

Scripture, E.W. (1902): The elements of experimental phonetics (New York, Charles Scribner's Sons/London, Arnold; 2. Aufl. Ams Press, New York 1973).

Sotavalta, Arvo (1936): Die Phonetik und ihre Beziehungen zu den Grenzwissenschaften (Annales Academiae Scientiarum Fennicae, B 31,3, Helsinki).

Straka, Georges (1963): La division des sons du langage en voyelles et consonnes peut-elle être justifiée? Travaux de Linguistique et de Littérature 1, 17-99.

Trubetzkoy, N. S. (1962): Grundzüge der Phonologie (Vandenhoeck \& Ruprecht, Göttingen, 2. Aufl.).

Zwirner, E. und Zwirner, Kurt (1966): Grundfragen der Phonometrie (Karger, Basel, 2, Aufl.). 


\section{Povzetek \\ LINGVISTIČNA FONETIKA}

Pričujoče delo skuša pokazati, kako bi se dalo eksperimentalno fonetiko kot lingvistično stroko popolnoma vključiti $v$ jezikoslovje. Pisec meni, da imajo fonetične raziskave smisel le tedaj, če je njihova problematika lingvistično usmerjena. Žal ni bilo vedno tako, zato je bil položaj fonetike $v$ jezikoslovju občasno negotov in tudi danes ni jasno opredeljen. Lingvistična fonetika mora izhajati iz fonologije kot svoje podlage. Ločitev fonetike od fonologije je torej po piščevem mnenju izključena. Fonetika in fonologija sta le dve strani iste stroke.

Dalje pisec svari pred težnjami, da bi zanemarili ali celo zanikali realnost fizikalnih oziroma fizioloških manifestacij jezika, npr. zaradi fonološke ideologije. Tak odnos ne koristi znanosti, temveč ustvarja nepremične bojne črte, te pa škodijo, saj otežujejo ali onemogočajo dialog med znanstveniki. 